\title{
Increase in cases of Guillain-Barré syndrome during a Chikungunya outbreak, French Polynesia, 2014 to 2015
}

E Oehler ${ }^{1}$, E Fournier ${ }^{2}$, I Leparc-Goffart ${ }^{3}$, P Larre ${ }^{4}$, S Cubizolle ${ }^{4}$, C Sookhareea 4 , S Lastère 5 , F Ghawche 4

1. Internal Medicine Department, French Polynesia Tertiary Hospital, Tahiti, French Polynesia

2. Clinical Neurophysiology Department, Pitié Salpétrière, APHP, Paris, France

3. French National Reference Centre for Arboviruses, IRBA, Marseille, France

4. Neurology Department, French Polynesia Tertiary Hospital, Tahiti, French Polynesia

5. Clinical Virology Laboratory, French Polynesia Tertiary Hospital, Tahiti, French Polynesia

Correspondence: Erwan Oehler (erwan.oehler@cht.pf)

Citation style for this article:

Oehler E, Fournier E, Leparc-Goffart I, Larre P, Cubizolle S, Sookhareea C, Lastère S, Ghawche F. Increase in cases of Guillain-Barré syndrome during a Chikungunya outbreak, French Polynesia, 2014 to 2015. Euro Surveill. 2015;20(48):pii=30079. DOI: http://dx.doi.org/10.2807/1560-7917.ES.2015.20.48.30079

Article submitted on 24 November 2015 / accepted on 03 December 2015 / published on 03 December 2015

During the recent chikungunya fever outbreak in French Polynesia in October 2014 to March 2015, we observed an abnormally high number of patients with neurological deficit. Clinical presentation and complementary exams were suggestive of GuillainBarré syndrome (GBS) for nine patients. All nine had a recent dengue-like syndrome and tested positive for chikungunya virus (CHIKV) in serology or RT-PCR. GBS incidence was increased four- to nine-fold during this period, suggesting a link to CHIKV infection.

Between October 2014 and March 2015, an estimated 66,000 cases of chikungunya virus infections were reported in French Polynesia, with an overall attack rate of $25 \%$ [1]. At the same time, we observed an epidemiological cluster of cases Guillain-Barré syndrome (GBS), an acute and probably autoimmune demyelinating polyradiculoneuropathy that appears after a triggering (viral or bacterial) infection in almost two thirds of cases. GBS had already been associated with several arboviral diseases including chikungunya virus (CHIKV) infections [2,3], but a cluster as we reported here had never been described.

\section{Cluster description}

Among the reported chikungunya fever cases, ca 50 people developed complications (myocarditis, meningoencephalitis, GBS) and 18 died; most of them had comorbidities. Nine patients were admitted for GBS based on clinical assessment, in the French Polynesia tertiary hospital: three in November, three in December, two in January and one in February. Six patients were male (male/female ratio: 2), with a median age of 48 years (range: $37-77$ years). Eight patients were Polynesian and one was Caucasian from Metropolitan France. Two patients had hypertension and one had hypertension and diabetes mellitus; the six others did not have any underlying conditions.
All nine were referred and then admitted to the department of neurology with a median length of stay of 11 days (range: 6-21 days). The patients were hospitalised within a median of eight days (range: 3-40 days) after the onset of symptoms of infection. Upon admission none had any symptoms of this acute phase which had presented as a dengue-like syndrome: all patients were febrile, eight had arthromyalgia, and three had a rash. GBS, presenting as a sensorimotor deficit beginning in the lower limbs and evolving to the upper limbs, was reported for eight patients; the last patient only had facial diplegia with sensory disorders of the face. Seven patients presented with signs of cranial nerve involvement, facial paralysis, dysphonia, dysarthria or dysphagia, associated with severity of GBS [4]. Among them, four were hospitalised in the intensive care unit for a median length of stay of six days (range: 2-15 days).

\section{Diagnostic findings in Guillain-Barré syndrome patients}

Eight patients had IgM and IgG antibodies against CHIKV and one was positive for CHIKV in RT-PCR positive. Serological evidence of past dengue or Zika virus infection ( $I g G)$ was found in nine and eight patients, respectively, IgM for both infections was negative in all cases; no PCR was done for Zika virus. No PCR was performed for dengue virus either but when performed, it was negative.

Brain and spinal magnetic resonance imaging (MRI) was performed in seven patients within a median of nine days (range: 5-15 days) after the onset of neurological symptoms. Among the seven patients presenting with cranial nerves involvement, MRI showed signs of neuritis of the facial nerves in two patients and neuritis of the facial and left trigeminal nerves in one patient; no abnormalities were seen in the others. MRIs which disclosed signs of neuritis were performed at least eight 
days after the beginning of GBS; those whose MRI was normal had it at day 5 and day 6; two patients only had a computerised tomography brain scan without contrast enhancement.

Electromyography was performed in all nine patients within a median of seven days (range: 3-14 days) after the start of GBS. It disclosed significant prolongation of the motor distal latencies with reduction of distal motor amplitudes, which attested a severe and predominant impairment of motor conduction in distal part of the nerves; motor conduction of more proximal nerve segments was less affected and little sensory impairment was seen.

All patients underwent a lumbar puncture, which was performed within a median of six days after the onset of neurological deficit (range: 2-15 days). Cerebrospinal fluid (CSF) was characterised by an albuminocytologic dissociation (elevated total protein concentration without CSF cell count abnormality) in all cases; median spinal protein concentration was $1.26 \mathrm{~g} / \mathrm{L}$ (range: 0.82-4.97; norm <0.5), whereas the median cell count was $2 / \mathrm{mm}^{3}$ (range: $1-6$; norm $<5$ ). Glucose levels were normal, with a median CSF/plasma ratio of 0.6 (range: 0.43-0.70; norm <0.75); CSF lactate levels were slightly elevated at a median of $167 \mathrm{mg} / \mathrm{L}$ (range: 117-251; norm <190). Plasma level of antiganglioside antibodies was measured in eight patients (anti-GM1, GM2, GD1a, GD1b, anti GQ1b IgM and IgG antibodies); a low positivity of anti-GM2 was noted in one case and of anti-GD1a in another case.

All patients were treated with intravenous immunoglobulin. Six patients were discharged to a functional rehabilitation centre for a median stay of 28 days (range: $19-60$ days). In all patients, electrophysiological parameters quickly returned to almost normal levels within less than three months.

\section{Discussion}

Chikungunya fever is an arboviral disease that usually manifests as a self-liming dengue-like syndrome with high fever, severe arthralgias and myalgias, and a maculopapular rash. Rare but severe complications may occur, such as myocarditis, hepatitis and neurological manifestations [5].

Neurological tropism of CHIKV seems to be lower than of other arboviruses such as dengue, West Nile or yellow fever viruses, but several studies have described, especially during epidemics, neurological manifestations such as meningoencephalitis, seizures or GBS [6]. In some cases, IgM antibodies against CHIKV have been found in CSF of patients with meningitis, supporting the theory of neuroinvasion [7].

While the annual incidence of GBS in French Polynesia is between 1 and 2 per 100,000 inhabitants, nine cases of GBS were admitted to our hospital during this sixmonth outbreak of chikungunya, a four- to nine-fold increased incidence, leading us to suspect a causal relationship between CHIKV infection and GBS. To our knowledge, this is the first series of GBS temporally associated with a chikungunya fever outbreak. Characteristics of the neurological presentation of our patients are not different from GBS related with, or following other aetiologies. We thus recommend keeping in mind that GBS is not an uncommon possibility in neurological disorders associated with CHIKV virus infection, especially in an epidemic context. Finally, it is interesting to note that this is the second arbovirustriggered outbreak of GBS in French Polynesia within two years: the first one, during the Zika fever outbreak from 2013 to 2014, resulted in 42 cases and a 20 -fold increase in the annual incidence of GBS [8].

Our report adds to the mounting body of evidence about the possibility of severe neurological disease following CHIKV infections.

\section{Conflict of interest}

None declared.

Authors' contributions

EO, SL, EF, ILG wrote the manuscript. EO, PL, SC, CS, FG took part in the clinical management of patients during the outbreak. SL collaborated in molecular biology techniques. ILG collaborated on the virological investigation. EF collaborated on the neurophysiological investigation. All authors read and approved the final manuscript.

\section{References}

1. Nhan TX, Musso D. The burden of chikungunya in the Pacific. Clin Microbiol Infect. 2015;21(6):e47-8. DOI: 10.1016/j. cmi.2015.02.018 PMID: 25749562

2. Lebrun G, Chadda $K$, Reboux $A H$, Martinet 0 , Gaüzère $B A$. Guillain-Barré syndrome after chikungunya infection.Emerg Infect Dis. 2009;15(3):495-6. DOI: 10.3201/eid1503.071482 PMID: 19239775

3. Villamil-Gómez W, Silvera LA, Páez-Castellanos J, RodriguezMorales AJ. Guillain-Barré syndrome after Chikungunya infection: A case in Colombia.Enferm Infecc Microbiol Clin. 2015. DOI: 10.1016/j.eimc.2015.05.012 PMID: 26164263

4. Bhargava A, Banakar BF, Pujar GS, Khichar S. A study of Guillain-Barré syndrome with reference to cranial neuropathy and its prognostic implication. J Neurosci Rural Pract. 2014;5(Suppl 1):43-7.

5. Lemant J, Boisson V, Winer A, Thibault L, André H, Tixier F, et al. Serious acute chikungunya virus infection requiring intensive care during the Reunion Island outbreak in 20052006. Crit Care Med. 2008;36(9):2536-41. DOI: 10.1097/ CCM.obo13e318183f2d2 PMID: 18679124

6. Lewthwaite P, Vasanthapuram R, Osborne JC, Begum A, Plank JL, Shankar MV, et al. Chikungunya virus and central nervous system infections in children, India. Emerg Infect Dis. 2009;15(2):329-31. DOI: 10.3201/eid1502.080902 PMID: 19193287

7. Chusri S, Siripaitoon P, Hirunpat S, Silpapojakul K. Case reports of neuro-Chikungunya in southern Thailand.Am J Trop Med Hyg. 2011;85(2):386-9. DOI: 10.4269/ajtmh.2011.10-0725 PMID: 21813863

8. Oehler $E$, Watrin L, Larre $P$, Leparc-Goffart I, Lastère S, Valour $\mathrm{F}$, et al. Zika virus infection complicated by Guillain-Barre syndrome--case report, French Polynesia, December 2013. Euro Surveill. 2014;19(9):20720. DOI: 10.2807/1560-7917. ES2014.19.9.20720 PMID: 24626205 